Revue bibliographique pour le domaine irano-aryen

\title{
Pierre Briant. « Peut-on parler d'«art perse»? Les interprétations de Georges Perrot et Charles Chipiez (1890) dans le contexte de leur élaboration »
}

\section{Reinhardt Pirngruber}

\section{(2) OpenEdition}

\section{Journals}

Electronic version

URL: https://journals.openedition.org/abstractairanica/53449

DOI: $10.4000 / a b s t r a c t a i r a n i c a .53449$

ISSN: 1961-960X

Publisher:

CNRS (UMR 7528 Mondes iraniens et indiens), Éditions de l'IFRI

Electronic reference

Reinhardt Pirngruber, "Pierre Briant. «Peut-on parler d'«art perse»? Les interprétations de Georges Perrot et Charles Chipiez (1890) dans le contexte de leur élaboration »", Abstracta Iranica [Online], Volume 42-43 | 2021, document 53, Online since 30 December 2021, connection on 23 December 2022. URL: http://journals.openedition.org/abstractairanica/53449 ; DOI: https://doi.org/10.4000/ abstractairanica. 53449

This text was automatically generated on 23 December 2022.

All rights reserved 


\title{
Pierre Briant. « Peut-on parler d'«art perse»? Les interprétations de Georges Perrot et Charles Chipiez (1890) dans le contexte de leur élaboration »
}

\author{
Reinhardt Pirngruber
}

\section{REFERENCES}

Pierre Briant. « Peut-on parler d'«art perse»? Les interprétations de Georges Perrot et Charles Chipiez (1890) dans le contexte de leur élaboration » in R. Rollinger, K. Ruffing, L. Thomas (eds.). Das Weltreich der Perser. Rezeption - Aneignung - Verargumentierung. Wiesbaden: Harrassowitz Verlag, 2019, p. 67-80.

1 P. Briant illuminates an early attempt at writing the art history of ancient Persia by two French scholars. After a brief introduction to the sources available to this early study, lauding the diligence of Perrot and Chipiez, the author shows that nevertheless they held Persian art in low esteem compared to its Near Eastern predecessors Egypt and Assyria and especially contemporary Greek art. Rather, it was judged a rather uninspiring synthesis of these different elements serving the sole purpose of glorifying the Great King. 


\section{AUTHORS}

\section{REINHARDT PIRNGRUBER}

Institut für Orientalistik, Wien 